\title{
Expondo os benefícios dos microrganismos aos seres humanos para alunos de Ensino
}

\section{Médio em São Luís-MA}

\author{
Exposing the benefits of micro-organisms to human beings for High School students in São Luís-MA \\ Exposición de los beneficios de los microorganismos a los seres humanos para estudiantes de
} escuela Secundaria en São Luís-MA

Recebido: 22/10/2021 | Revisado:30/10/2021 | Aceito: 03/11/2021 | Publicado: 07/11/2021

\author{
Beatriz Gomes Vila Nova \\ ORCID: https://orcid.org/0000-0001-5403-8520 \\ Universidade Ceuma, Brasil \\ E-mail: beatrizgvilanova@hotmail.com \\ Lucas dos Santos Silva \\ ORCID: https://orcid.org/0000-0002-7483-3238 \\ Universidade Ceuma, Brasil \\ E-mail: 1s.luscas@gmail.com \\ Rafaella Santos Sabóia \\ ORCID: https://orcid.org/0000-0001-6190-0262 \\ Universidade Ceuma, Brasil \\ E-mail: rafa_saboia@hotmail.com \\ Lully Gabrielly Silva Alves \\ ORCID: https://orcid.org/0000-0003-3655-5115 \\ Universidade Ceuma, Brasil \\ E-mail: lullygaby@hotmail.com \\ Paulo Dyago Borges Gomes \\ ORCID: https://orcid.org/0000-0002-5926-8939 \\ Universidade Ceuma, Brasil \\ E-mail: pauloborges.99@outlook.com \\ Lívia Câmara de Carvalho Galvão \\ ORCID: https://orcid.org/0000-0001-9540-649X \\ Universidade CEUMA, Brasil \\ E-mail: livia.camara@ceuma.br \\ Eduardo Martins de Sousa \\ ORCID: https://orcid.org/0000-0002-2506-169X \\ Universidade CEUMA, Brasil \\ E-mail: eduardo.martins@ceuma.br
}

\begin{abstract}
Resumo
Tratar dos microrganismos e os relacionar com o seu extenso leque de benefícios, no âmbito escolar, é de suma importância na perspectiva de deixar-se de lado o conceito de que os mesmos são apenas nocivos à saúde humana. $\mathrm{O}$ objetivo do projeto foi averiguar o que estudantes do ensino médio compreendem ou acreditam compreender sobre os microrganismos, e a partir disso, promover conhecimento e desfazer os conceitos distorcidos, assegurando que os microrganismos também são passíveis de benefícios. O projeto foi realizado no Colégio Marista Araçagy localizado no município de São José de Ribamar, no Maranhão, com alunos do $3^{\circ}$ ano do ensino médio. Previamente os alunos foram pré-avaliados para que pudéssemos estimar seus conhecimentos acerca dos microrganismos e quão benéficos são. Logo em seguida o tema foi abordado com o auxílio de slides, onde definiu-se conceitos, benefícios e exemplos foram relatados. Seguidamente, aplicou-se novamente um questionário com o intuito de mensurarmos o rendimento e aprendizado após os sujeitarmos à ação proposta. Após análise do questionário, verificou-se que dos 31 alunos que participaram, 39\% não tinham conhecimento sobre a temática, enquanto $61 \%$ disseram já ter tido contato com o tema e $10 \%$ dos estudantes afirmaram que todas as bactérias são patogênicas. Só foi possível evidenciarmos um cenário animador após avaliarmos as respostas do segundo questionário. Contudo, favorecer conhecimento prévio sobre os microrganismos e seus benefícios é sem dúvida primordial na construção do saber acerca do que é verídico.
\end{abstract}

Palavras-chave: Ensino básico; Microbiologia; Microrganismo; Probióticos.

\section{Abstract}

Dealing with microorganisms and relating them to their extensive range of benefits, in the school environment, is of paramount importance in the perspective of putting aside the concept that they are only harmful to human health. The aim of the project was to find out what high school students understand or believe they understand about microorganisms, and from that, promote knowledge and undo distorted concepts, ensuring that microorganisms are 
also likely to benefit. The project was carried out at the Colégio Marista Araçagy public school located in the municipality of São José de Ribamar, in Maranhão, with students from the $3^{\circ}$ year of high school. Previously, the students were pre-evaluated so that we could estimate their knowledge about microorganisms and how beneficial they are. Soon after, the theme was approached with the help of slides, where concepts, benefits and examples were reported. Then, a questionnaire was applied again in order to measure the performance and learning after subjecting them to the proposed action. After analyzing the questionnaire, it was found that of the 31 students who participated, $39 \%$ had no knowledge about the topic, while $61 \%$ said they had already had contact with the topic and $10 \%$ of the students said that all bacteria are pathogenic. It was only possible to evidence an encouraging scenario after evaluating the responses to the second questionnaire. However, favoring prior knowledge about microorganisms and their benefits is undoubtedly essential in the construction of knowledge about what is true.

Keywords: Basic education; Microbiology; Microorganism; Probiotics.

\section{Resumen}

Tratar con los microorganismos y relacionarlos con su amplia gama de beneficios en el entorno escolar es de suma importancia en la perspectiva de dejar de lado el concepto de que solo son perjudiciales para la salud humana. El objetivo del proyecto era averiguar qué entienden o creen los estudiantes de secundaria sobre los microorganismos y, a partir de eso, promover el conocimiento y deshacer conceptos distorsionados, asegurando que los microorganismos también se beneficien. El proyecto se llevó a cabo en el Colégio Marista Araçagy ubicado en el municipio de São José de Ribamar, en Maranhão, con estudiantes de 3er año de secundaria. Previamente, los estudiantes fueron evaluados previamente para poder estimar su conocimiento sobre los microorganismos y cuán beneficiosos son. Poco después, se abordó el tema con la ayuda de diapositivas, donde se reportaron conceptos, beneficios y ejemplos. Luego, se les aplicó nuevamente un cuestionario con el fin de medir el desempeño y el aprendizaje luego de someterlos a la acción propuesta. Luego de analizar el cuestionario, se encontró que de los 31 estudiantes que participaron, el 39\% no tenía conocimiento sobre el tema, mientras que el $61 \%$ dijo que ya había tenido contacto con el tema y el $10 \%$ de los estudiantes dijo que todas las bacterias son patógenas. Solo fue posible evidenciar un escenario alentador luego de evaluar las respuestas al segundo cuestionario. Sin embargo, favorecer el conocimiento previo sobre los microorganismos y sus beneficios es sin duda fundamental en la construcción del conocimiento sobre la verdad.

Palabras clave: Educacion basica; Microbiología; Microorganismo; Probióticos.

\section{Introdução}

A microbiologia é caracterizada pelo estudo do papel dos microrganismos relacionado a sociedade, à saúde humana e ao meio ambiente (Staink, 2013). Assim, a microbiologia trata temas sobre a natureza básica e prática, podendo ser usada como fontes de produtos e aplicações importantes. Compreende-se ainda a sua ecologia, como esses organismos microscópicos vivem e interagem no ecossistema, evidenciando seus malefícios e possíveis benefícios para a humanidade (Madigan, et al., 2016).

Os microrganismos são por vezes apresentados como agentes causadores de diversas doenças, conceituando-os exclusivamente como prejudicais a saúde humana (Kimura, et al., 2013). Essa situação pode desencadear confusão e incerteza nos conceitos formados por muitos estudantes quando tiverem contato com o estudo dos microrganismos (Freitas, et al., 2018). Dessa forma, é evidente a necessidade de desenvolver conteúdos voltados para a microbiologia capazes de mostrar aplicações benéficas e importantes da mesma, a fim de mostrar suas utilidades e despertar interesse dos alunos (Cândido, et al., 2015).

Existem inúmeros microrganismos que estabelecem relações benéficas com o corpo humano, por exemplo a microbiota, que consiste um conjunto de variados microrganismos presentes no corpo humano, contribuindo na digestão de alimentos, produção de vitaminas e proteção contra patógenos (Ceribeli, et al., 2017). Além disso, pode-se observar no cotidiano importantes funções realizadas no meio ambiente por meio da ação dos microrganismos, tais como: processo de decomposição, reciclagem de elementos químicos (carbono, nitrogênio, enxofre, fósforo e outros), tratamento de esgoto e também produção de alimentos e outros (Tortora, et al., 2012).

$\mathrm{Na}$ indústria de alimentos utiliza-se várias aplicações microbiológicas, destaca-se o processo de fermentação realizado por fungos e bactérias, principalmente as do gênero Lactobacillus, esses microrganismos são importantes para o crescimento de pães, produção de iogurtes, vinagres, bebidas alcoólicas, queijos e entre outros (Bernardi, et al., 2019). Certos alimentos contendo bactérias fermentadoras de ácido láctico, como alguns laticínios e iogurtes, representam uma fonte de 
microrganismos que podem regular de forma benéfica a saúde intestinal e até mesmo tratar ou prevenir doenças inflamatórias intestinais (Jung, et al., 2018). Com base nessas propriedades, os alimentos enriquecidos com esses microrganismos moduladores são denominados probióticos (Santos, et al., 2020).

Probióticos são definidos como "microrganismos vivos" que, quando administrados em quantidades adequadas conferem um benefício à saúde no hospedeiro. $\mathrm{O}$ uso de probióticos e seus benefícios são amplamente conhecidos com utilização em distúrbios intestinais, doença cardíaca coronária, alergia e prevenção do câncer (Jun-mo \& Yoo, 2017). Tem-se demonstrado também aplicações consideráveis desses microrganismos na fabricação de vacinas, medicamentos (por exemplo, antibiótico penicilina) e na biotecnologia (Tortora, et al., 2017). Enzimas produzidas por esses microrganismos também tem sido utilizada e manipulada para uso de circunstâncias terapêuticas, como na produção de insulina (Barreto \& Silva, 2020).

Outro processo com uso de microrganismos é a biorremediação que tem ganhado bastante destaque para o tratamento de impactos ambientais (Leonel, et al., 2018). Nessa técnica faz-se o uso de microrganismos afim de degradar ou transformar o contaminante em substâncias menos nocivas para o meio ambiente contaminado (Mallmann, et al., 2019).

Dessa forma, buscar informações sobre o entendimento dos alunos à cerca da microbiologia é essencial para que incertezas e coisas da mesma natureza sejam esclarecidas e para que estabeleça uma relação mais próxima entre o estudo dos microrganismos e os alunos, não tendo mais dúvidas do quão benéficos os microrganismos podem ser (Ferreira, 2010).

Portanto, submeter os estudantes à novos conceitos e despertar o interesse deles por algo que já existe, mas que não é do seu conhecimento é imprescindível para que eles possam refletir e dá significado a esses novos conhecimentos a respeito dos microrganismos. Assim, o objetivo desse trabalho é mostrar os benefícios que os microrganismos podem proporcionar aos seres humanos.

\section{Metodologia}

\section{1 Área de atuação}

Realizou-se uma pesquisa quali-quantitativa, de caráter exploratório, em campo, onde é observados todo os fatos e como eles acontecem. A partir dos questionamentos aplicados é possível estudar todas as relações estabelecidas. Trata-se de uma pesquisa qualitativa porque busca estudar todos os tópicos aplicados, com ações bastante ampla, e buscando avaliar como os entrevistados veem e quais seus conhecimentos diante do conteúdo estudado, neste caso, foram aplicados questionário para os alunos. Caracteriza-se quantitativa, pois foi determinado por meio de números todo o conhecimento avaliado para que fossem identificados e analisados (Pereira, et al., 2018).

Foi realizado na escola de rede privada Colégio Marista Araçagy localizada no município de São José de Ribamar Maranhão, tendo como público-alvo os alunos do $3^{\circ}$ ano do ensino médio. Todas as atividades ocorreram nas dependências da escola. Com mais de 90 anos de fundação, o colégio é referência, da educação infantil ao ensino médio, e faz parte de uma rede internacional de colégios, presente em 82 países. No Brasil, está nas cinco regiões brasileiras. Atualmente, no país, a rede é composta por 20 Colégios, 9 Escolas Sociais, mais de 30 mil estudantes, mais de 5 mil colaboradores.

Em 13 de novembro de 2004, o Marista prossegue sua atuação na Ilha de São Luís, agora no município de São José de Ribamar, como Colégio Marista Araçagy. Inicialmente com o prédio do Educação Infantil (Maristinha). Em 2006, foi inaugurado o bloco do Ensino Fundamental e Ensino Médio (Maristão). O Colégio Marista do Araçagy, com 16 anos de atuação, está em constante movimento, sempre buscando os melhores recursos a fim de aprimorar os espaços que já possui. Desde 2019, destaca-se alguns feitos: inauguração da Sala Maker, Sala de Robótica, Laboratório de Matemática, Laboratório de Ciências e reinauguração da Piscina Olímpica. O colégio tem sempre investido na renovação de diversos espaços e processos pedagógicos. 


\subsection{Ação estratégica}

De forma híbrida com parte dos alunos presente na sala e outra parte em casa via software Microsoft Teams, foi realizado a aplicação de um questionário (pré-teste) composto por algumas questões, divididas em duas partes sendo uma metade composta de perguntas objetivas (respostas sim ou não) e 4 perguntas de múltipla escolha (podendo marcar mais de uma opção, de um total de 5) com intuito de avaliar o conhecimento prévio dos alunos sobre a relação de benefícios que os microrganismos podem ter para com o ser humano.

Em seguida, a temática foi explorada através de uma palestra expositiva com a presença dos membros executores do projeto, e neste momento foram contempladas algumas funções dos microrganismos de importância para o homem, como fabricação de medicamentos, produção de alimentos, biorremediação, microbiota intestinal e probióticos. Por fim, aplicou-se outro questionário (pós-teste) a fim de medir-se o aprendizado e desempenho destes frente à ação proposta.

\subsection{Recursos (Humanos e físicos)}

A atividade foi realizada no dia 08 de outubro de 2021 e teve organização e execução pelos alunos do Programa de Mestrado em Biologia Microbiana da Universidade CEUMA, campus Renascença, São Luís-MA, tendo suas despesas financeiras - transporte, alimentação e fotocópias - arcadas por conta própria. Utilizou-se, ainda, recursos como datashow e computador, disponíveis no próprio colégio. A aprovação do projeto ocorreu mediante conversa inicial com a diretora da escola e, posteriormente, envio de ofício apresentando o projeto aos demais professores e coordenadores da escola.

\section{Resultados e Discussão}

Ao todo 31 alunos participaram da ação. Na pré e pós avaliação todos os alunos receberam questionários, os estudantes fisicamente presentes na palestra receberam o questionário impresso e aos que estavam assistindo a palestra de forma online foi disponibilizado um link de acesso. Abordou-se os participantes sobre o nível de conhecimento que eles tinham referente ao tema abordado na palestra (Figura 1).

Figura 1: Conhecimento prévio dos alunos a respeito do tema abordado.

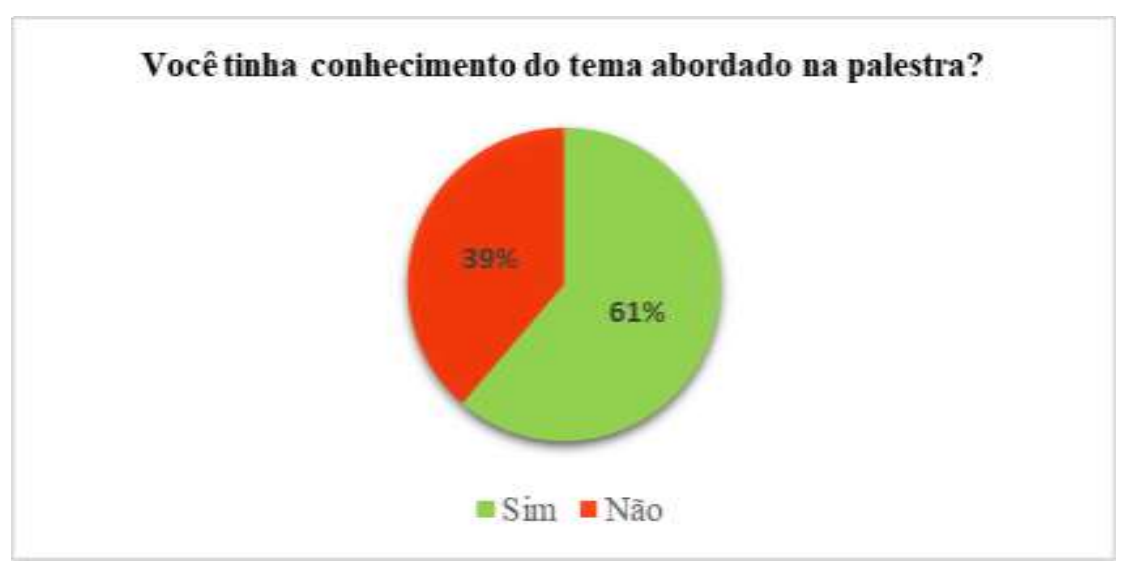

Fonte: Elaborado pelos autores.

Ao serem questionados acerca do conteúdo abordado, notou-se que $39 \%$ dos estudantes relataram não ter conhecimento, enquanto $61 \%$ disseram já ter tido contato com o tema. É fundamental que as compreensões prévias sobre os microrganismos e suas relações com a saúde humana e ambiente sejam identificadas. Essa identificação auxilia na elaboração de atividades que promovam a associação microbiologia-cotidiano (Zompero, 2009). A educação em microbiologia é um 
assunto extremamente necessário para desmitificação de ideias difundidas a respeito dos microrganismos, onde é possível proporcionar aprendizagem, mudanças conceituais, reflexões e transformar as condutas errôneas dos estudantes (Felix, et al., 2020).

O conhecimento sobre a microbiologia básica é um assunto bastante relevante e indispensável para construção dos estudantes como cidadãos conscientes e preparados a encarar a vida no seu dia a dia (Bezerra, et al., 2015). Visto que, é uma área prática relacionada à saúde e higiene pessoal de cada indivíduo, assim como, ligada a outras situações importantes para o funcionamento do meio ambiente (Ferreira, 2010).

No questionário aplicado antes da intervenção, 10\% dos estudantes afirmaram que todas as bactérias são patogênicas, portanto, fica evidente que nem todos os alunos tinham conhecimento das propriedades benéficas que esses seres podem trazer aos seres humanos. Todavia, após a realização da palestra todos os alunos mudaram seu ponto vista (Figura 2).

Figura 2: Questão pré e pós intervenção sobre todas as bactérias serem patogênicas.

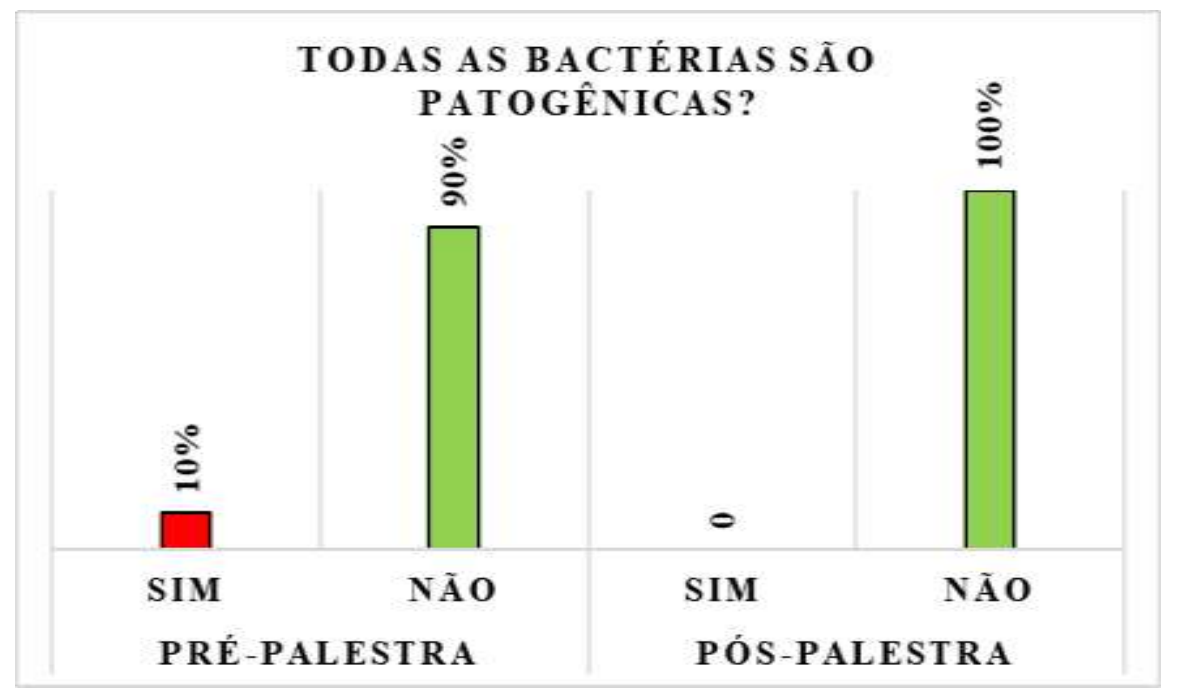

Fonte: Elaborado pelos autores.

Isso também é evidenciado na pesquisa de Freitas e colaboradores (2020), ao realizaram uma intervenção para avaliar o conhecimento prévio dos estudantes sobre o tema de microrganismos em geral. Posteriormente foi realizado uma aula teórico-prática com intuito de desvincular suas interpretações equivocadas sobre microrganismos. Após a intervenção foi visto concepções diferentes dos alunos e aprendizagem significativa. Outro trabalho desenvolvido por Oliveira, et al., (2016), demostraram que grande parte dos alunos também tem essa visão em que as bactérias são estritamente causadoras de doenças.

De fato, os microrganismos estão associados causadores de doenças, no entanto, a grande maioria não são totalmente maléficos para os seres humanos (Jacobucci \& Jacobucci, 2009). Em vista disso, precisa-se esclarecer perspectivas diferentes a respeito dos microrganismos aos estudantes e não somente apresentar nos seus aspectos negativos, mas também mostrar suas relações benéficas (Toledo, et al., 2015).

Outro importante dado observado foi que $48 \%$ dos alunos não souberam responder o que é a microbiota, além disso, $39 \%$ deles achavam que microbiota fosse o termo que designa todos os microrganismos que existem, 3\% achavam que eram todas as bactérias que causavam doenças e 6\% não souberam responder (Figura 3). Todavia, após a realização da palestra notou-se uma evolução e $94 \%$ dos estudantes já sabiam responder corretamente o que é microbiota (Figura 4). 
Figura 3: Questão pré-intervenção sobre microbiota.

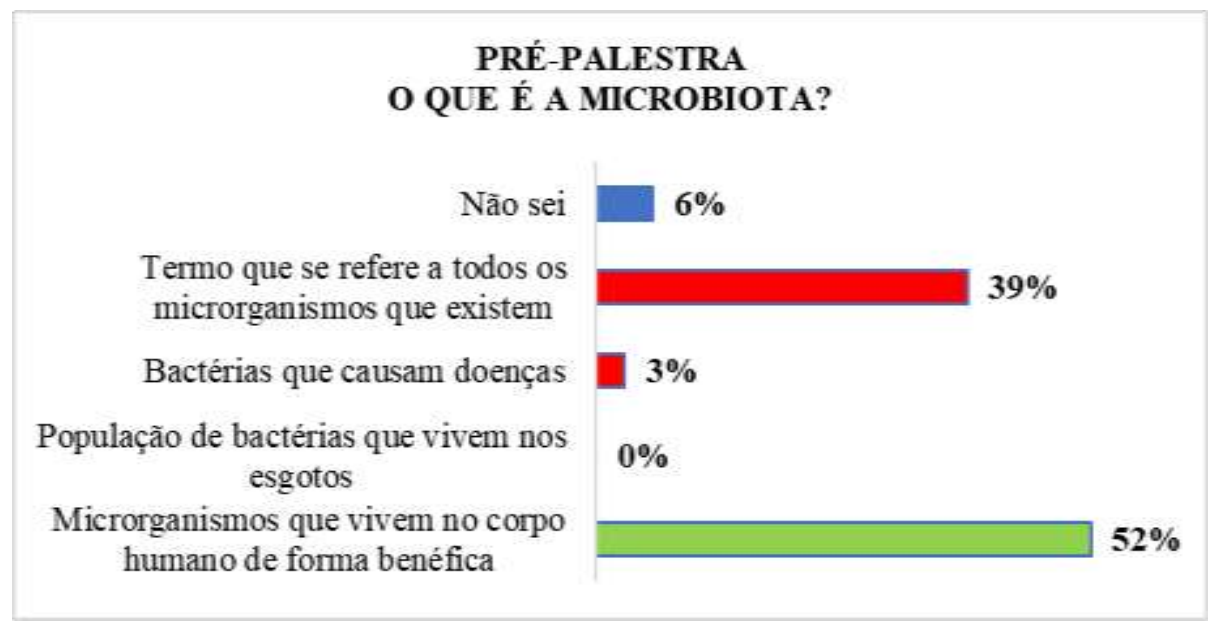

Fonte: Elaborado pelos autores.

Fica evidente que nem todos os alunos tem uma visão ampla a respeito dos microrganismos e que podem ser encontrados em qualquer lugar, até mesmo no corpo humano mantendo relações benéficas por meio da microbiota (Bernadi, et al., 2019). Mesmo sendo amplamente distribuído na terra, os microrganismos são considerados apenas potencialmente patogênicos e insignificantes por muitos (Madigan, et al., 2010).

Figura 4: Questão pós-intervenção sobre microbiota.

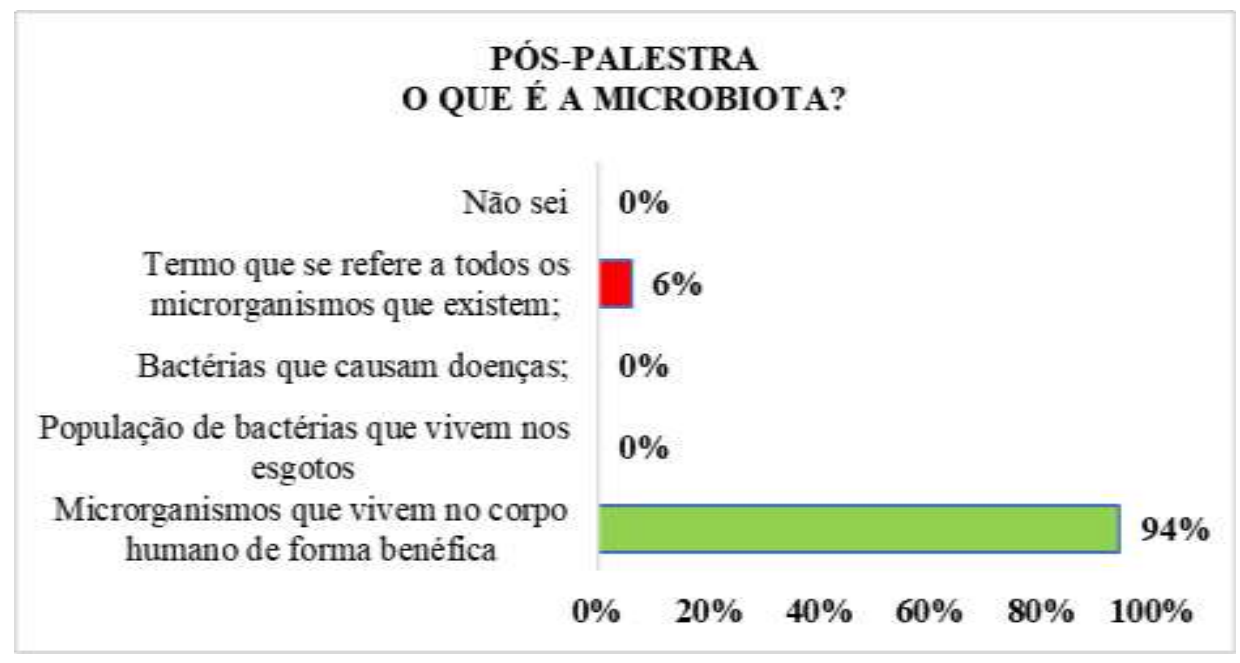

Fonte: Elaborado pelos autores.

Previamente, ao serem questionados sobre os probióticos quase metade dos estudantes (42\%) informaram não saber, contudo, após a realização da palestra interventiva todos os alunos, de forma unânime responderam corretamente à esta questão (Figura 5). 
Figura 5: Questão pré e pós intervenção sobre probióticos.

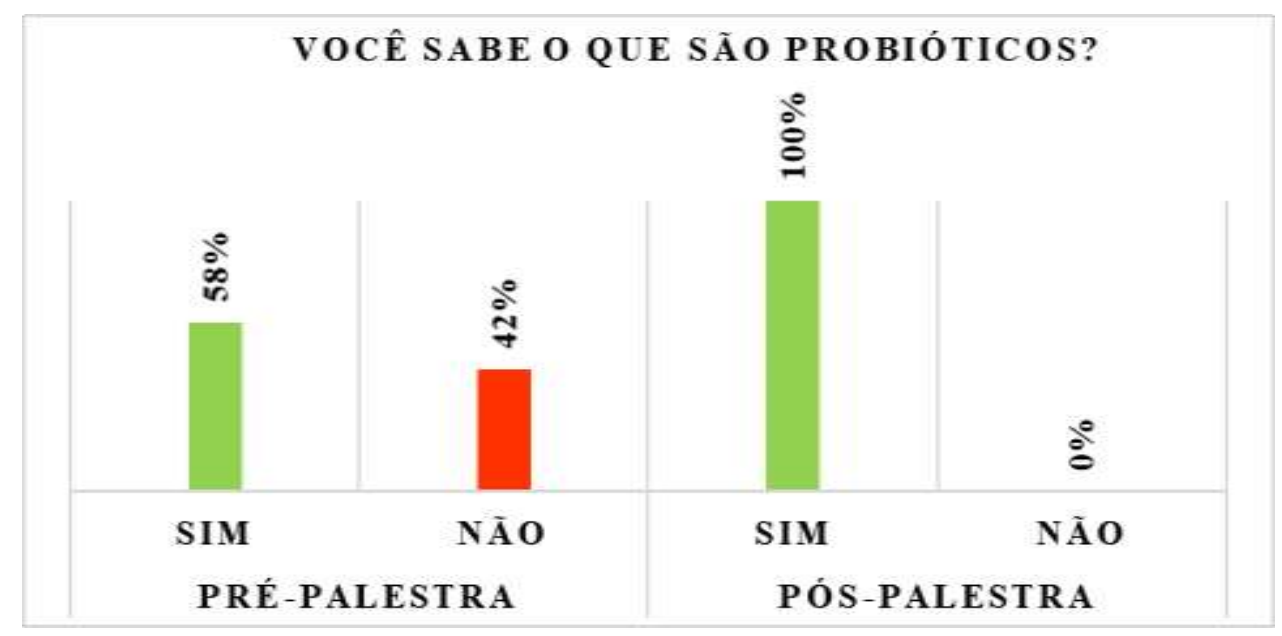

Fonte: Elaborado pelos autores.

Conceitos relacionados aos benefícios e uso de microrganismos na alimentação são poucos conhecidos. Sabe-se que existem inúmeros microrganismos importantes para a saúde do hospedeiro como os probióticos, que são responsáveis por promover o equilíbrio da microbiota normal (Oliveira, et al., 2017).

Após a intervenção da palestra as mesmas perguntas foram realizadas, e os resultados foram outros, mostrando o sucesso da proposta de intervenção. Diferentes trabalhos (Ferreira, 2010; Kimura, et al., 2013; Freitas, et al., 2020; Albuquerque, et al., 2012) demonstram que muitos alunos tem conceitos equivocados sobre os microrganismos e que após o processo de intervenção, os alunos obtiveram outra visão a respeito dos microrganismos.

\section{Conclusão}

Esta pesquisa propôs avaliar o conhecimento de estudantes do ensino médio de uma escola particular sobre os microrganismos, promovendo conhecimento e desfazendo os conceitos distorcidos e errôneos, assegurando que os microrganismos também são passíveis de benefícios. Considera-se que a palestra em conjunto com o questionário aplicado esclareceu características básicas acerca dos benefícios dos microrganismos para os seres humanos, mostrando que essa interatividade entre palestrantes e alunos foi capaz de expandir o interesse dos estudantes sobre aspectos microbiológicos básicos.

Quanto aos conceitos abordados referentes à microbiologia as análises foram positivas, uma vez que os alunos conseguiram responder questões de uma forma que antes não tinham conseguido sem a associação da palestra. Contudo, no geral, os resultados obtidos foram satisfatórios e demonstraram que a maior parte dos alunos possuem um bom conhecimento acerca do tema abordado. Ressalta-se ainda a importância de trabalhos como esse, que conseguem difundir o conhecimento acadêmico e levá-lo à sociedade de forma simples e prazerosa. Espera-se que propostas como essa sejam cada vez mais estimuladas e cheguem a atingir uma importante parcela da sociedade. Sugere-se a trabalhos futuros visar públicos mais carentes em conhecimento sobre o tema abordado, dando assim um impacto ainda maior na proposta interventiva.

\section{Referências}

Albuquerque, G. G., da Silva, R. P B., \& Gomes, V. (2012). Conhecimento dos alunos sobre microrganismos e seu uso no cotidiano. Revista de Educação, Ciências e Matemática, 2(1).

Bernardi, G., dos Santos Silveira, M., de Freitas Leonardi, A., Ferreira, S. A., \& Goldschmidt, A. I. (2019). Concepções prévias dos alunos dos anos iniciais sobre microrganismos. Revista Ciências \& Ideias ISSN: 2176-1477, 10(1), 55-69. 
Bezerra, A. C., Magalhães, A. D. S., Bordoni, C. V., Michiles, D. E. F., Aires, R. D. S., Souza, P. R. B. D., \& Santos, V. M. D. (2015). Trabalhando com microbiologia no ambiente escolar. Anais Programa Ciência Na Escola, 3(1).

Cândido, M. D. S. C., Santos, M. G., de Medeiros Azevedo, T., \& Neto, L. S. (2015). Microbiologia no ensino médio: analisando a realidade e sugerindo alternativas de ensino numa escola estadual paraibana. Ensino, Saúde e Ambiente, 8(1).

Ceribeli, C., Nitschke, M., \& Porto, A. L. (2017). Divulgando ciências para estudantes do nível fundamental: projeto de extensão em microbiologia. Enciclopédia biosfera, 14(25).

Feitas, P. N. N., Justus, J. F. C., Pileggi, S. A. V., \& Pileggi, M. (2020). Ressignificação de conceitos sobre microrganismos por meio de mapas conceituais em alunos de ensino médio. Experiências em Ensino de Ciências, 15(3), 410-430.

Freitas Zompero, A. (2009). Concepções de alunos do ensino fundamental sobre microorganismos em aspectos que envolvem saúde: implicações para o ensino aprendizagem. Experiências em Ensino de Ciências, 4(3), 25-37.

Oliveira, J. L., de Almeida, C., \& da Silva Bomfim, N. (2017). A importância do uso de probióticos na saúde humana. Unoesc \& Ciência-ACBS, 8(1), 7-12.

Felix, I. C. G., Silva-Júnior, L. C. F., Neto, R. D. M., da Costa Silva, P. J., Lima, J. V. M. R., Nunes, B. E. B. R., \& Duarte, A. W. F. (2020). Ensino de Microbiologia em escola pública: contribuições da extensão universitária na formação médica. Extensio: Revista Eletrônica de Extensão, 17(37), 108-123.

Ferreira, A. F. (2010). A importância da microbiologia na escola: uma abordagem no ensino médio. Rio de Janeiro.

Freitas, V. S., de Sá, C. A., \& de Castro, K. R. (2018). O ensino de microbiologia nas escolas públicas de ensino fundamental do município de Jaguaribe, Ceará. Conexões-Ciência e Tecnologia, 12(1), 84-96.

Jacobucci, D. F. C., \& Jacobucci, G. B. (2009). Abrindo o Tubo de Ensaio: o que sabemos sobre as pesquisas em Divulgação Científica e Ensino de Microbiologia no Brasil. JCOM, 8(2), 1-8.

Jung, C. E., Hochstedler, B. R., Price, T., Wolfe, A. J., \& Brubaker, L. (2018). The Urinary Microbiota of Women with Recurrent Urinary Tract Infections: Opportunities to Improve Clinical Care. PFD Week 2018.

Kim, J. M., \& Park, Y. J. (2017). Probiotics in the prevention and treatment of postmenopausal vaginal infections. Journal of Menopausal Medicine, 23(3), $139-145$.

Kimura, A. H., de Oliveira, G. S., Scandorieiro, S., de Souza, P. C., Schuruff, P. A., Medeiros, L. P., \& Kobayashi, R. K. T. (2013). Microbiologia para o ensino médio e técnico: contribuição da extensão ao ensino e aplicação da ciência. Revista Conexão UEPG, 9(2), 254-267.

Leonel, L. V., do Nascimento, E. G., Bertozzi, J., Bôas, L. A. V., \& Bôas, G. T. V. (2018). Biorremediação do solo. Revista Terra \& Cultura: Cadernos de Ensino e Pesquisa, 26(51), 37-52.

Madigan, M. T., Martinko, J. M., Bender, K. S., Buckley, D. H., \& Stahl, D. A. (2016). Microbiologia de Brock-14a Edição. Artmed Editora.

Madigan, M. T., Martinko, J. M., Dunlap, P. V., \& Clark, D. P. (2010). Microbiologia de Brock. (12a . Ed.) Editora Artmed, Porto Alegre, RS, 1160.

Mallmann, V., Aragão, L. W. R., Fernandes, S. S. L., Fernandes, T. C. L., Aragão, R. F. R., \& da Silva, R. C. D. L. (2019). As Vantagens da Biorremediação na Qualidade Ambiental. Ensaios e Ciência C Biológicas Agrárias e da Saúde, 23(1), 12-15.

Oliveira, N. F. D. (2014). Concepções alternativas sobre microorganismos: alerta para a necessidade de melhoria no processo ensino-aprendizagem de biologia.

Pereira, A. S., Shitsuka, D. M., Parreira, F. J., \& Shitsuka, R. (2018). Metodologia da pesquisa científica.

Santos, P. S., de Almeida, E. B., Lacerda, L. G., do Nascimento, L. C. G., \& Pereira, M. C. S. (2020). Consumo de probióticos e os benefícios para a saúde. Revista Cereus, 12(1), 2-15.

Staink, D. R. (2013). A ciência da microbiologia. Santa Maria: Disciplina de Microbiologia Geral. Santa Maria: Universidade Federal de Santa Maria.

Teixeira, J. J. D. O. (2017). Relatório de atividade profissional (Dissertação de doutorado).

Toledo, A. G., Poersch, K. M., do Nascimento, J. E., \& de Lima, B. G. T. (2015). Estudo da microbiologia e sua relação no cotidiano do aluno a partir da temática saúde. Ensino, Saude e Ambiente, 8(2).

Tortora, G. J., Case, C. L., \& Funke, B. R. (2017). Microbiologia-(12ª Edição.) Artmed Editora. 